\title{
A Static Characteristic Analysis of Proposed Bi-Directional Dual Active Bridge DC-DC Converter
}

\author{
Shun Nagata \\ Nagasaki University \\ 1-14 Bunkyo-machi \\ Nagasaki-shi, Nagasaki, Japan \\ bb52113224@cc.nagasaki-u.ac.jp
}

\section{Mika Takasaki}

\author{
Nagasaki University \\ 1-14 Bunkyo-machi \\ Nagasaki-shi, Nagasaki, Japan \\ bb52112218@cc.nagasaki-u.ac.jp
}

\section{Yutaka Furukawa}

Koga System Works

Saga, Japa

\section{Toshiro Hirose}

Nishimu Electronics Industries Co.,Ltd.

700 Tateno, Yoshinogari-cho

Kanzaki-gun, Saga, Japan

hirose@nishimu.co.jp

\begin{abstract}
Recently, the power supply network with energy storage devices such as battery has been focused. This network topology uses bi-directional isolated DC-DC converter of low or medium capacity is required for the diversification of power supply network. The dual active bridge (DAB) DC-DC converter is one of the effective bi-directional isolated DC-DC converters. However, the circuit has some instinct problems such as degradation of power efficiency and the occurrence of the surge in light-load operation. In this paper, we have been done a static characteristic analysis and highly power-efficient technique for DAB DC-DC Converter at light load. Also the analysis results and the proposed technique are verified with some experimental results.
\end{abstract}

\section{INTRODUCTION}

Recently, the bidirectional dc-dc converter has been focused on because of the huge demand for diversification of power supply network including battery. The DAB dc-dc converter is one of the most popular circuits for bidirectional applications because of its simple structure. Some examples are for UPS [1], for automotive [2]-[4] and for energy storage system [5]. The one of the feature is achieving zero volt switching (ZVS) in natural operation. However, hard switching and/or power efficiency at light load condition is the intrinsic problem [6]. Some research have been done to solve the problem, for instance, use of resonant type converter with snubber circuit [7], silicon carbide (SiC) power device and new magnetic materials [8], and QuasiZCS operation with LC filter [9]. Furthermore by applying switching modulation, DAB converter works in wide range of input voltage and load condition [10]-[12]. These objectives of switching modulation controls are to regulate voltage and satisfy load variation [10], to expand soft switching region [11], and minimize the total power losses [12]. However, the problem of switching surges reduction was not addressed. In [13], the novel switching surge reduction technique is proposed and confirmed with some analysis and experimental results. And also, the results of power efficiency improvement of the light load were described.

In this paper, the detailed analysis of the technique is described and confirmed with some experiments.

\section{CONVENTIONAL OPERATION OF A DAB DC-DC CONVERTER}

Fig. 1 shows the circuit schematic of the basic DAB dc-dc

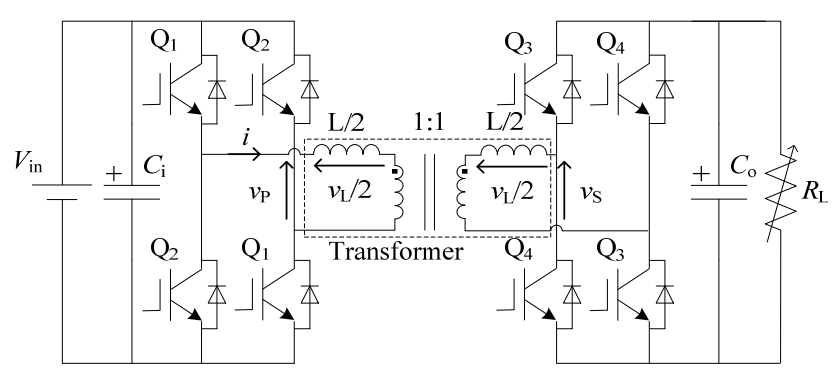

Fig. 1. The circuit schematic of DAB dc-dc converter. 


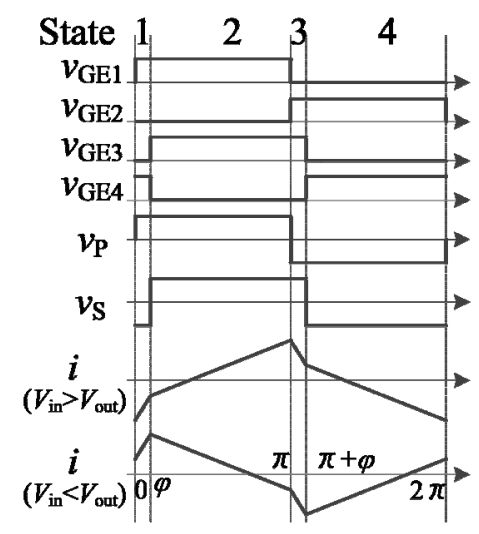

(a) Light load

Fig. 2.

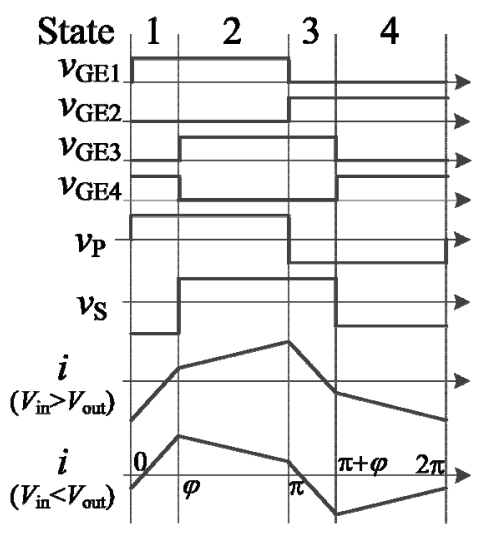

(b) Heavy load

converter. Fig. 2 shows the operating waveforms with the conventional operation [14]. In the conventional operation, the output power is operated by the phase-shift shown as $\varphi$ between the primary voltage $v_{\mathrm{P}}$ and secondary voltage $v_{\mathrm{S}}$ of transformer. Fig. 3 shows the phasor diagram. $V_{\mathrm{P}}, V_{\mathrm{S}}, V_{\mathrm{L}}$, and $I$ are phasor symbols for $v_{\mathrm{P}}, v_{\mathrm{S}}, v_{\mathrm{L}}, i$, respectively. When $V_{\mathrm{S}}$ is lagging $V_{\mathrm{P}}$ in forward power flow mode (Fig. 3 (a) and (b)), and when $V_{\mathrm{S}}$ is leading $V_{\mathrm{P}}$, it is operated in reverse power flow mode (Fig. 3 (c)).

The output power $P_{\mathrm{o}}$ can be obtained as

$$
P_{o}=\frac{V_{\text {in }} V_{\text {out }}}{\omega L} \varphi\left(1-\frac{\varphi}{\pi}\right) \text {. }
$$

The output power can be controlled with the phase difference $\varphi$. The waveform of the current $i$ is changed by the load condition. In this paper, current $i$ crossed the zero line in the state 2 is defined as a light load, and current $i$ crossed the zero line in the state 1 is defined as a heavy load as shown in Fig. 2.

\section{INTRINSIC SURGES PROBLEM OF A DAB DC-DC CONVERTER}

As mentioned in above, well known problem of a DAB DC-DC converter is hard switching in the light condition. However, previous researches haven't been addressed about the switching surges problem. It is caused by the reverse recovery effect of the diode. Fig. 4 shows $\varphi-P_{\mathrm{o}}$. The switching surges occur at light load range of this figure.

Fig. 5 shows the generation mechanism of switching surges when $V_{\text {in }}>V_{\text {out }}$. The surges voltage occurs in the transition from State 1 (3) to State 2 (4), repeatedly. $C_{\mathrm{d}}$ is the parasitic capacitance of diode which is connected in parallel with the ideal diode, and $L_{\text {wire }}$ is parasitic reactance. At the light load condition, the diodes $\mathrm{D}_{4}$ is conducting in state 1 . Then the switches $\mathrm{Q}_{3}$ is turned on when state changes from State 1 to State 2. At this instantaneous moment, the diode $\mathrm{D}_{4}$ is switched from a forward bias condition to a reverse bias condition, immediately. And the switching surges are

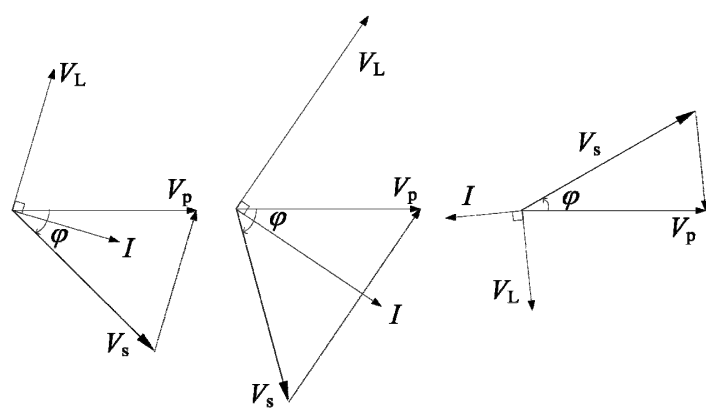

(a)

(b)

(c)

Fig. 3. Phasor diagram [13]: (a) Forward power flow mode (light load); (b) Forward power flow mode (heavy load); (c) Reverse power flow mode.

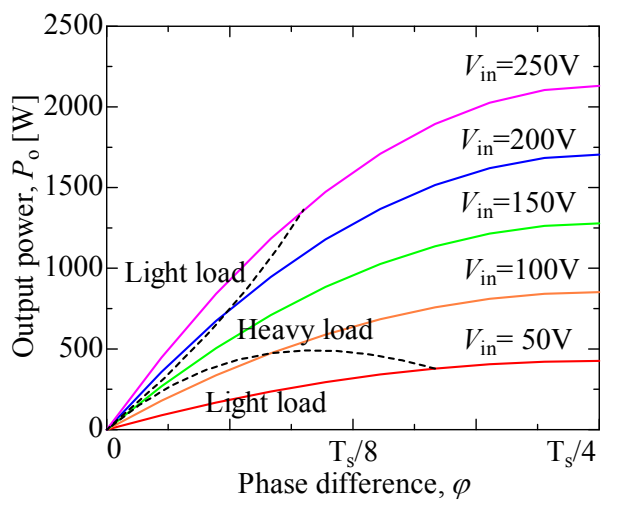

Fig. 4. $\varphi-P_{\mathrm{o}}\left(V_{\mathrm{in}}=150 \mathrm{~V}\right)[13]$.

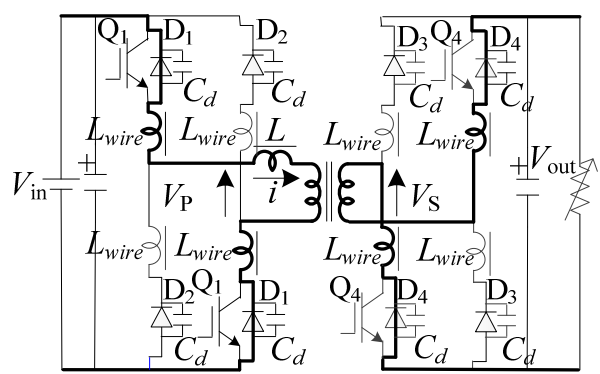

(a) State 1

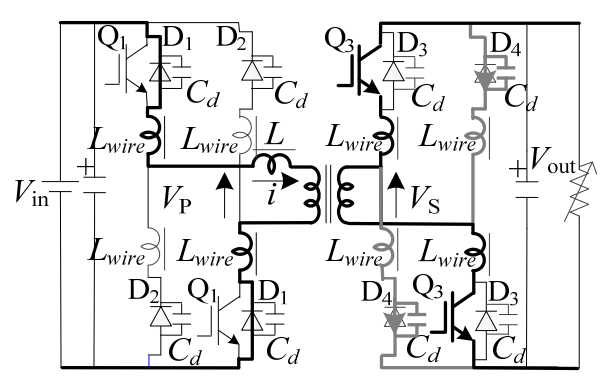

(b) State 2

Fig. 5. The generation mechanism of switching surge $\left(V_{\text {in }}>V_{\text {out }}\right)$ 


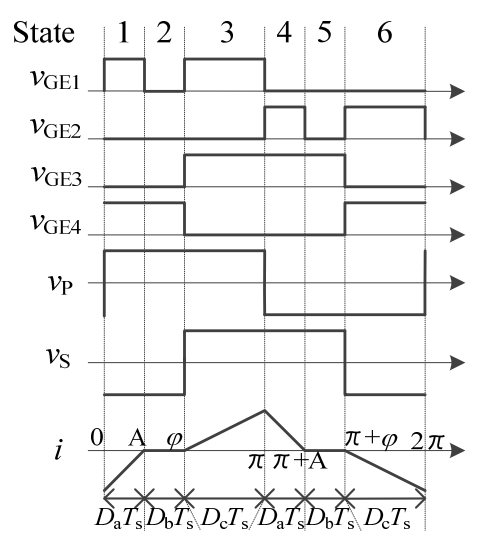

(a) Buck mode

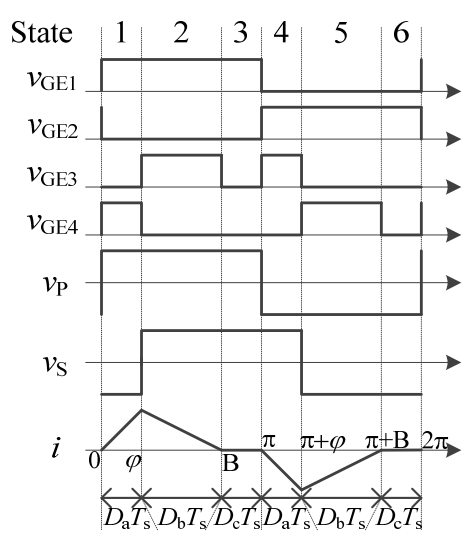

(b) Boost mode

Fig. 6. Idealized waveforms for proposed operating at the light load.

occurred with the resonance of $C_{\mathrm{d}}$ and $L_{\text {wire }}$ due to reverse recovery phenomenon. With the same reason, when $V_{\text {in }}<V_{\text {out }}$, the surges occurs in the transition from State 2 (4) to State 3 (1) on the primary side.

Commonly, to protect the switches from the switching surges, snubber circuit are applied. However, the power loss at the snubber circuit can't be ignored at the light load condition. The other way, the resonant converter type is also popular, but the additional components are needed [9].

\section{Proposed Operation Method}

We have proposed the software-based compensation method for basic DAB dc-dc converter topology. It can be reduce the switching surges at the light load, without any of additional circuits such as the snubber circuits or resonant circuits [13]. Fig. 6 shows idealized waveform of the proposed operating method. When $V_{\text {in }}<V_{\text {out }}$, as it can be seen from the waveforms, the direction of primary side current of transformer $i$ during each on-time of $\mathrm{Q}_{1}$ and $\mathrm{Q}_{2}$ is restricted to avoid the crossing the zero line. Due to the restriction, the zero-current-switching can be realized for $Q_{1}$ and $Q_{2}$, respectively. The ideal static analysis has been done as follows. This converter has six operational states in one switching period for each of the buck and boost mode operation, respectively. The each element is treated as ideal in equivalent circuit.

The detailed description of the ideal circuit is revealed in a previous paper [13]. Therefore, only the results are shown in this paper.

\section{A. Buck Mode Operation in Light Load}

In buck mode, the primary side switches $\mathrm{Q}_{1}$ and $\mathrm{Q}_{2}$ are turned-on twice in the period. Firstly, $\mathrm{Q}_{1}$ and $\mathrm{Q}_{2}$ are turn-on at $t=0$ and $T_{\mathrm{s}} / 2$. Secondly, they are turn-off at $t=A$ and $T_{\mathrm{s}} / 2$ $+A$. Thirdly, they are turn-on at $t=\varphi$ and $T_{\mathrm{s}} / 2+\varphi$. Fourthly, they are turn-off at $t=T_{\mathrm{s}} / 2$ and $T_{\mathrm{s}}$.

$A$ is calculated as

$$
A=\frac{V_{\text {in }}-V_{\text {out }}}{V_{\text {in }}+V_{\text {out }}}\left(\frac{1}{2} T_{s}-\varphi\right) .
$$

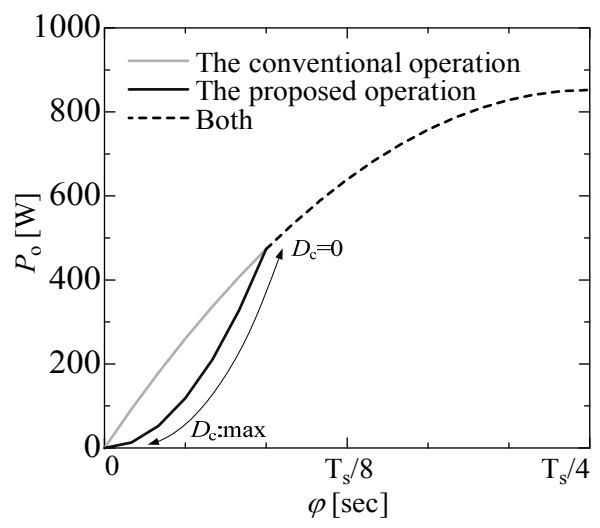

(a) Buck mode

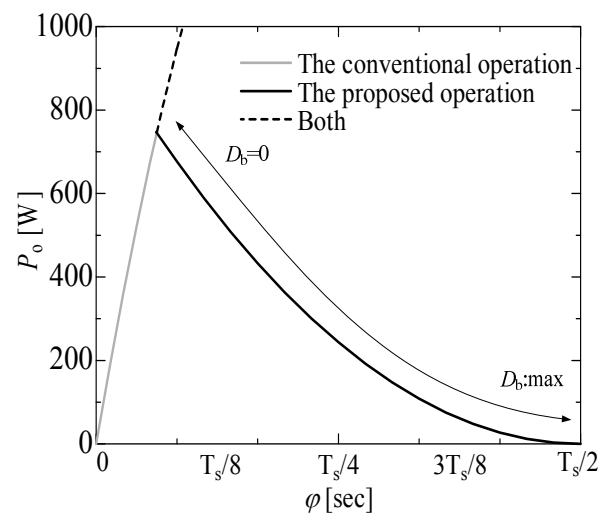

(b) Boost mode

Fig. 7. $\varphi-P_{\mathrm{o}}$ (conventional operation and proposed operation).

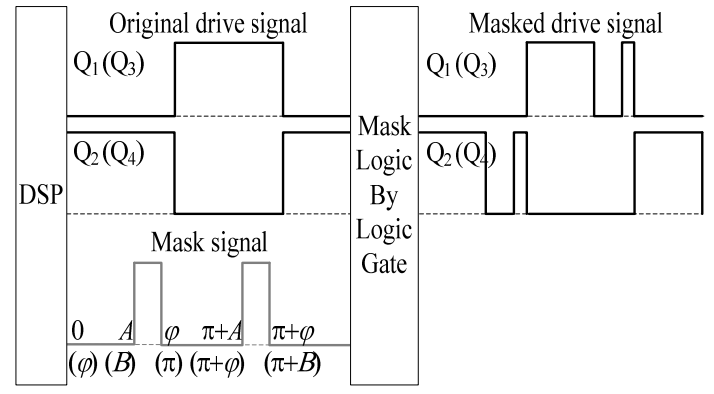

Fig.8. Masked drive signal generating mechanism.

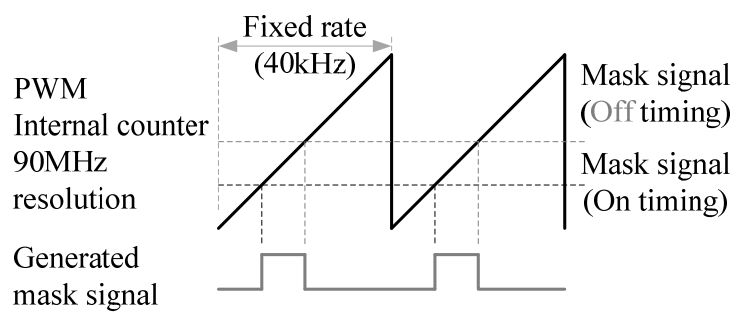

Fig. 9. Mask signal generating mechanism of PWM in DSP. 


\section{B. Boost Mode Operation in Light Load}

In boost mode, the secondary side switches $\mathrm{Q}_{3}$ and $\mathrm{Q}_{4}$ are turned-on twice in the period. Firstly, $\mathrm{Q}_{3}$ and $\mathrm{Q}_{4}$ are turn-on at $t=\varphi$ and $T_{\mathrm{s}} / 2+\varphi$, respectively. Secondly, they are turn-off at $t=B$ and $T_{\mathrm{s}} / 2+B$. Thirdly, they are turn-on at $t=T_{\mathrm{s}} / 2$ and 0 . Fourthly, they are turn-off at $t=T_{\mathrm{s}} / 2+\varphi$ and $\varphi$.

$B$ is calculated as

$$
B=\frac{2 V_{\text {out }}}{V_{\text {out }}-V_{\text {in }}} \varphi
$$

\section{Output Power Control in Light Load}

The ideal analysis for both of buck and boost mode operation can be done for power. For the ideal analysis result, the output power $P_{\mathrm{o}}$ can be obtained as

$$
P_{o}=\frac{2 X^{2}}{T_{s} L} \cdot\left|\frac{V_{\text {in }}+V_{\text {out }}}{V_{\text {in }}-V_{\text {out }}}\right| \cdot V_{\text {in }} V_{\text {out }} .
$$

In buck mode, $X=A$, and in boost mode, $X=\varphi$.

\section{Output Power Control in Heavy Load}

In the light load, with the output power increasing, the periods of which all switches turned OFF $(A \sim \varphi, \pi+A \sim \pi+$ $\varphi, B \sim \pi, \pi+B \sim 2 \pi$ ) becomes shorter. $A$ equal to $\varphi$ or $B$ equal to $\pi$ is the boundary between light load and heavy load. Therefore, in the heavy load condition, the only conventional phase-shift operation is active. From the results, it can be seen that it is possible to control the output power seamlessly despite of the load condition. Relationship $\varphi$ and $P_{\mathrm{o}}$ of conventional and proposed operation is shown in Fig. 7.

\section{E. Pulse Generating Method}

Fig. 8 and Fig. 9 show the generating mechanism of proposed driving signal. As mentioned above, the gate signal is the combination of the phase shift signal and the masked signal. The mask width is calculated and controlled by (2) and (3), respectively.

\section{LOSS INCLUDED ANALYSIS OF CONVENTIONAL OPERATION}

Equation of the output power (1) was equation for the ideal state without consideration of the conduction loss of the body diode and switch and the parasitic resistance of the transformer. This chapter will be described analysis of static characteristics in consideration of these losses. In order to analyze and make some definitions, in the operation waveform of Figure 2, both of light load and heavy load can be divided into four states. Since the basic operation of two half cycles are symmetric, only the first half cycle is explained. To analyze the characteristics of the circuit, Extended State-Space Averaging Method [16] is applied.

In order to simplify the loss analysis, loss is defined as $r_{\text {loss. }}$ Equivalent circuits corresponding to each state in buck mode operation are shown in Fig.9, where $\hat{v}_{o}$ is the low-frequency component of $V_{\mathrm{o}}$.

For analysis, solving for $i_{\mathrm{L}}$ and $i_{\mathrm{c}}$,

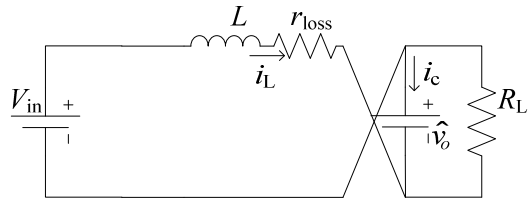

(a) state 1

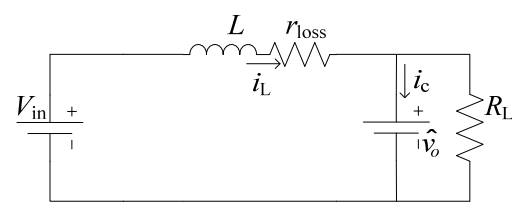

(b) state2.

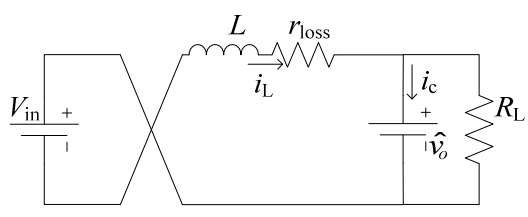

(c) state3.

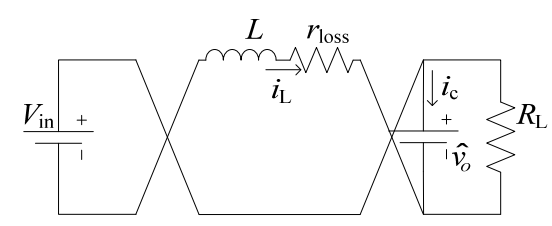

(d) state 4 .

Figure 9. Equivalent circuit :

(a) state 1; (b) state 2; (c) state 3; (d) state 4;

for $0 \leq t \leq D T_{\text {s }} \quad$ (state 1$)$

In Fig. 9(a), Voltage law of the circuit is

$$
L \frac{d i_{L 1}(t)}{d t}=V_{\text {in }}+\hat{v}_{\text {out }}-i_{L 1}(t) r_{\text {loss }} .
$$

Integration of eq.(5) is

$$
L \cdot i_{L 1}(t)=\int\left(V_{i}+v_{o}\right) d t-r_{\text {loss }} \int i_{L 1}(t) d t+C .
$$

Linear approximation of eq.(6) is

$$
\int i_{L 1}(t) d t=\frac{1}{2}\left\{i_{L 1}(0)+i_{L 1}(t)\right\} t .
$$

Using eq.(5) and (6),

$$
i_{L 1}(t) \approx \frac{2\left(V_{i}+v_{o}\right) t}{2 L+r_{\text {loss }} t}+\frac{2 L-r_{\text {loss }} t}{2 L+r_{\text {loss }} t} \cdot i_{L 1}(0) .
$$

In addition, the current law is expressed as

$$
i_{c 1}(t)=-i_{L 1}(t)-\frac{\hat{v}_{\text {out }}}{R_{L}} .
$$

for $D T_{s} \leq t \leq \pi \quad$ (state 2)

Solving for current law and voltage law of circuit in the same way 


$$
\begin{aligned}
& i_{L 2}(t) \approx \frac{2\left(V_{i}-v_{o}\right)\left(t-D T_{s}\right)}{2 L+r_{\text {loss }}\left(t-D T_{s}\right)} \cdot \\
&+\frac{2 L-r_{\text {loss }}\left(t-D T_{s}\right)}{2 L+r_{\text {loss }}\left(t-D T_{s}\right)} \cdot\left\{\frac{2\left(V_{i}+v_{o}\right) D T_{s}}{2 L+r_{\text {loss }} D T_{s}}+\frac{2 L-r_{\text {loss }} D T_{s}}{2 L+r_{\text {loss }} D T_{s}} i_{L 1}(0)\right\} \\
& i_{c 2}(t)=i_{L 2}(t)-\frac{\hat{v}_{\text {out }}}{R_{L}} .
\end{aligned}
$$

In one cycle in the steady state, the current flowing through the leakage inductance is positive and negative symmetry operation. The operation of the state 3 and State 4 is equivalent to positive and negative symmetry to the operation of the state 1 and state 2 Therefore, the analysis was performed only for half cycle.

Since the conventional operation of two half cycles are symmetric,

$$
i_{L 1}(0)=-i_{L 2}\left(\frac{1}{2} T_{s}\right)
$$

It is possible to determine the initial value of the circuit using the eq. (12).

$i_{L 1}(0) \approx-\frac{2\left(V_{i}-v_{o}\right) \cdot\left(2 L+r_{\text {loss }} \cdot D T_{s}\right)(1-2 D) T_{s}+2\left(V_{i}+v_{o}\right)\left\{4 L-r_{\text {loss }} \cdot(1-2 D) T_{s}\right\} \cdot D T_{s}}{\left\{4 L+r_{\text {loss }} \cdot(1-2 D) T_{s}\right\}\left(2 L+r_{\text {loss }} \cdot D T_{s}\right)+\left\{4 L-r_{\text {loss }} \cdot(1-2 D) T_{s}\right\}\left(2 L-r_{\text {loss }} \cdot D T_{s}\right)}$

Next, deriving for the average current in the output capacitor of the state 1 and state2.The average value of $v$ in each state is calculated with

$i_{c 1_{-} \text {ave }} \approx-\frac{\left(V_{i}+v_{o}\right) \cdot D^{2} T_{s}^{2}}{2 L+r_{\text {loss }} \cdot D T_{S}}+\left(1+\frac{2 L-r_{\text {loss }} \cdot D T_{S}}{2 L+r_{\text {loss }} \cdot D T_{S}}\right)$.

$\frac{\left(V_{i}-v_{o}\right) \cdot\left(2 L+r_{\text {loss }} \cdot D T_{s}\right)(1-2 D) D T_{s}^{2}+\left(V_{i}+v_{o}\right)\left\{4 L-r_{\text {loss }} \cdot(1-2 D) T_{s}\right\} \cdot D^{2} T_{s}^{2}}{\left.\left.\left(4 L+r_{s}\right) \cdot(1-2 D) T_{s}\right)\left(2 L+r_{b s}\right)+D T\left(2 L-T_{s}\right) \cdot D T_{s}\right)}$

$\left\{4 L+r_{\text {loss }} \cdot(1-2 D) T_{s}\right\}\left(2 L+r_{\text {loss }} \cdot D T_{s}\right)+\left\{4 L-r_{\text {loss }} \cdot(1-2 D) T_{s}\right\}\left(2 L-r_{\text {loss }} \cdot D T_{s}\right)$ $-\frac{\hat{v}_{o}}{R_{L}} \cdot D T_{S}$

$i_{c 2_{-a v e}} \approx \frac{1}{2} \frac{\left(V_{i}-v_{o}\right)(1-2 D)^{2} T_{s}^{2}}{4 L+r_{\text {loss }}(1-2 D) T_{s}}+\frac{1}{2} \frac{\left(V_{i}+v_{o}\right) \cdot(1-2 D) D T_{s}^{2}}{2 L+r_{\text {loss }} \cdot D T_{s}}\left[1+\frac{4 L-r_{\text {loss }}(1-2 D) T_{s}}{4 L+r_{\text {loss }}(1-2 D) T_{s}}\right]$

$-\frac{1}{2}\left[1+\frac{4 L-r_{\text {loss }}(1-2 D) T_{s}}{4 L+r_{\text {loss }}(1-2 D) T_{s}}\right] \frac{2 L-r_{\text {loss }} \cdot D T_{s}}{2 L+r_{\text {loss }} \cdot D T_{s}}$.

$\frac{\left(V_{i}-v_{o}\right) \cdot\left(2 L+r_{\text {loss }} \cdot D T_{s}\right)(1-2 D)^{2} T_{s}^{2}+\left(V_{i}+v_{o}\right)\left\{4 L-r_{\text {loss }} \cdot(1-2 D) T_{s}\right\} \cdot(1-2 D) D T_{s}^{2}}{\left\{4 L+r_{\text {loss }} \cdot(1-2 D) T_{s}\right\}\left(2 L+r_{\text {loss }} \cdot D T_{s}\right)+\left\{4 L-r_{\text {loss }} \cdot(1-2 D) T_{s}\right\}\left(2 L-r_{\text {loss }} \cdot D T_{s}\right)}-\frac{\hat{v}_{o}}{R_{L}} \cdot \frac{1-2 D}{2} T_{s}$

while $\alpha=2 \omega L+r_{\text {loss }} \varphi, \beta=2 \omega L-r_{\text {loss }} \varphi, \gamma=4 \omega L+2 r_{\text {loss }}(\pi-\varphi)$ and

$\lambda=4 \omega L-2 r_{\text {loss }}(\pi-\varphi)$.

The results of static characteristics are obtained by letting $d \hat{v}_{o} / d t=0\left(\right.$ therefore $\left.\overline{i_{c_{o}}}=C d \hat{v}_{o} / d t=0\right)$.

The output power $P_{o}$ is

$$
P_{o} \approx V_{i} V_{o} \varphi\left(1-\frac{\varphi}{\pi}\right)\left\{\begin{array}{l}
\frac{1}{\pi-\varphi}\left(1+\frac{V_{o}}{V_{i}}\right)\left[\frac{\varphi \lambda}{\alpha \gamma+\beta \lambda}+\left(1-\frac{\beta \lambda}{\alpha \gamma+\beta \lambda}\right)\left\{\frac{\lambda}{\alpha \gamma}(\pi-\varphi)+\frac{1}{\alpha}(\pi-2 \varphi)\right\}\right] \\
+\left(1-\frac{V_{o}}{V_{i}}\right)\left[\frac{8 \omega L}{\alpha \gamma+\beta \lambda}+\frac{2 \pi}{\varphi}\left\{\frac{1}{\gamma}\left(1-\frac{\beta \lambda}{\alpha \gamma+\beta \lambda}\right)-\frac{\beta}{\alpha \gamma+\beta \lambda}\right\}\left(1-\frac{\varphi}{\pi}\right)\right]
\end{array}\right\} \text {. }
$$

When the $r_{\text {loss }}=0$ in equation (16), is found to be obtained the same equation as the equation (1). Fig.10 shows the characteristics of changing the value of $r_{\text {loss }}$. We can see the effect of $r_{\text {loss }}$ on the output power Po by Fig. 10 .

\section{LOSS INCLUdED ANALYSIS OF PROPOSED OPERATION}

To analyze the characteristics of the circuit, Extended

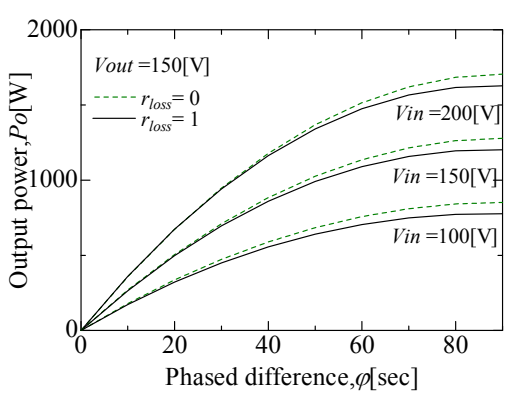

Figure 10. $\varphi-P$ o

(conventional analysis and proposed analysis).

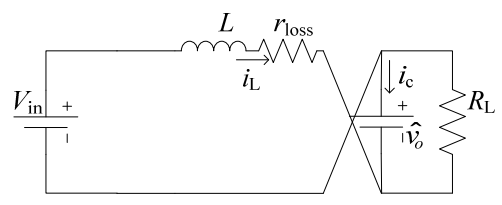

(a) State 1

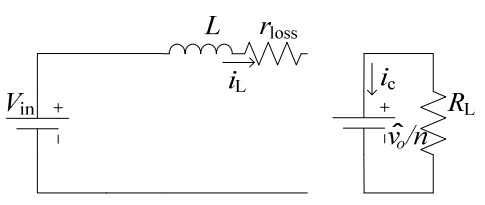

(b) State 2

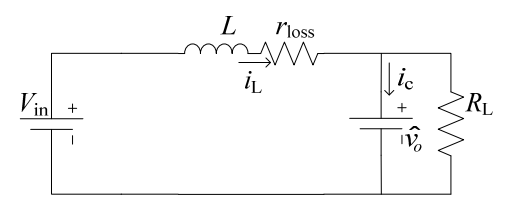

(c) State 3

Fig. 11. Equivalent circuit of buck mode operation.

State-Space Averaging Method [16] is applied again.

The analysis has been done for each of buck mode and boost mode operation, respectively. In order to simplify the loss analysis, loss is defined as $r_{\text {loss. }}$.

\section{A. Buck Mode Operation}

Equivalent circuits corresponding to each state in buck mode operation are shown in Fig. 11, where $\hat{v}_{o}$ is the lowfrequency component of $V_{\mathrm{o}} . D_{\mathrm{a}}=(\mathrm{A}-0) / T_{\mathrm{s}}, D_{\mathrm{b}}=(\varphi-\mathrm{A}) / T_{\mathrm{s}}$, $D_{\mathrm{c}}=(\pi-\varphi) / T_{\mathrm{s}}$ in Fig. 6 (a). For ease of analysis, the calculation has been performed in a half of the switching period because of the symmetric behavior of the circuit topology.

For analysis, solving for $i_{\mathrm{L}}$ and $i_{\mathrm{c}}$, for $0 \leq t \leq A$ (state 1$)$

$$
i_{L} \approx \frac{2\left(V_{\text {in }}+\hat{v}_{o}\right) t}{2 L+r_{\text {loss }} t}-\frac{2 L-r_{\text {loss }} t}{2 L+r_{\text {loss }} t} i_{L}(0)
$$




$$
i_{C_{o}}=-i_{L}-\frac{\hat{v}_{o}}{R_{L}}
$$

for $A \leq t \leq \varphi$ (state 2)

$$
\begin{gathered}
i_{L}=0 \\
i_{C_{o}}=-i_{L}-\frac{\hat{v}_{o}}{R_{L}}
\end{gathered}
$$

for $\varphi \leq t \leq \pi$ (state 3$)$

$$
\begin{gathered}
i_{L} \approx \frac{2\left(V_{i n}-\hat{v}_{o}\right)\left\{t-\left(D_{a}+D_{b}\right) T_{s}\right\}}{2 L+r_{\text {loss }}\left\{t-\left(D_{a}+D_{b}\right) T_{s}\right\}} \\
i_{C_{o}}=i_{L}-\frac{\hat{v}_{o}}{R_{L}} .
\end{gathered}
$$

From Fig. 6 , it is clear that $D_{\mathrm{a}}+D_{\mathrm{b}}+D_{\mathrm{c}}=1 / 2, i_{\mathrm{L}}(0)=-i_{\mathrm{L}}(\pi)$ and $i_{\mathrm{L}}(A)=0$. Using the preceding relationships,

$$
i_{L}(0)=-i_{L}(\pi)=-\frac{2\left(V_{i n}+\hat{v}_{o}\right) D_{a} T_{s}}{2 L-r_{\text {loss }} D_{a} T_{s}}
$$

and

$$
D_{c}=\frac{V_{i n}+\hat{v}_{o}}{V_{\text {in }}-\hat{v}_{o}-2 D_{a} T_{s} V_{\text {in }} r_{\text {loss }} / L} D_{a} .
$$

The average value of $i$ in each state is calculated with

$$
\begin{gathered}
i_{c_{c_{-}} \text {avel }}=-\frac{1}{2} \frac{2\left(V_{\text {in }}+\hat{v}_{o}\right) D_{a} T_{s}}{2 L-r_{\text {loss }} D_{a} T_{s}}-\frac{\hat{v}_{o}}{R_{L}} \\
i_{c_{o_{-}} \text {ave } 2}=-\frac{\hat{v}_{o}}{R_{L}} \\
i_{c_{c_{-} \text {ave }}=-\frac{1}{2}} \frac{2\left(V_{\text {in }}+\hat{v}_{o}\right) D_{a} T_{s}}{2 L-r_{\text {loss }} D_{a} T_{s}}-\frac{\hat{v}_{o}}{R_{L}} .
\end{gathered}
$$

Hence,

$$
\begin{array}{r}
\overline{i_{c_{o}}}=2\left(i_{c_{o-} \text { ave1 }} \times D_{a}+i_{c_{o-a} \text { ave } 2} \times D_{b}+i_{c_{o-a} \text { ave } 3} \times D_{c}\right) \\
=\frac{2 D_{a}^{2} T_{s}}{L} \cdot \frac{V_{i n} \hat{v}_{o}\left(V_{\text {in }}+\hat{v}_{o}\right)}{V_{\text {in }}-\hat{v}_{o}-V_{\text {in }} D_{a} T_{s} r_{\text {loss }} / L}-\frac{\hat{v}_{o}}{R_{L}} .
\end{array}
$$

The results of static characteristics are obtained by letting $d \widehat{v}_{o} / d t=0$. And using $D_{\mathrm{a}} T_{\mathrm{s}}=A$,

$$
P_{o}=\frac{2 A^{2}}{T_{s} L} \cdot \frac{V_{\text {in }} V_{\text {out }}\left(V_{\text {in }}+V_{\text {out }}\right)}{V_{\text {in }}-V_{\text {out }}-V_{\text {in }} D_{a} T_{s} r_{\text {loss }} / L}
$$

where

$$
A=\frac{\left(V_{\text {in }}-V_{\text {out }}\right)\left(T_{s} / 2-\varphi\right)}{V_{\text {in }}+V_{\text {out }}+V_{\text {in }}\left(T_{s} / 2-\varphi\right) r_{\text {loss }} / L} .
$$

\section{B. Boost Mode Operation}

Equivalent circuits corresponding to each state in boost mode operation are shown in Fig. 12. For analysis, equation is formularized for each state. $D_{\mathrm{a}}=(\varphi-0) / T_{\mathrm{s}}, D_{\mathrm{b}}=(B-\varphi) / T_{\mathrm{s}}$ and $D_{\mathrm{c}}=(\varphi-B) / T_{\mathrm{s}}$ in Fig. 6 (b).

For $0 \leq t \leq \varphi$ (state 1$)$

$$
\begin{gathered}
i_{L} \approx \frac{2\left(V_{\text {in }}+\hat{v}_{0}\right) t}{2 L+r_{\text {loss }} t} \\
i_{C_{o}}=-i_{L}-\frac{\hat{v}_{o}}{R_{L}}
\end{gathered}
$$

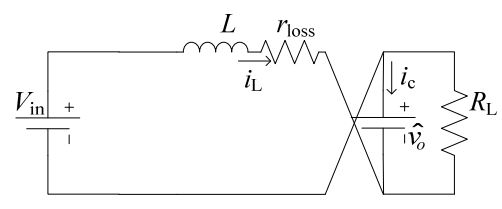

(a) State 1

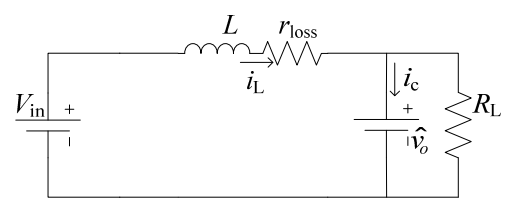

(b) State 2

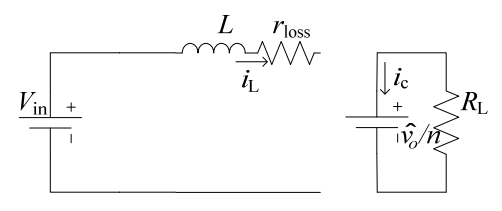

(c) State 3

Fig. 12. Equivalent circuit of boost mode operation.

for $\varphi \leq t \leq B$ (state 2)

$$
\begin{gathered}
i_{L} \approx \frac{2\left(V_{\text {in }}-\hat{v}_{0}\right)\left(t-D_{a} T_{s}\right)}{L+r_{\text {loss }}\left(t-D_{a} T_{s}\right)}+\frac{2 L-r_{\text {loss }}\left(t-D_{a} T_{s}\right)}{2 L+r_{\text {loss }}\left(t-D_{a} T_{s}\right)} i\left(D_{a} T_{s}\right) \\
i_{C_{o}}=i_{L}-\frac{\hat{v}_{o}}{R_{L}}
\end{gathered}
$$

for $B \leq t \leq \pi$ (state 3$)$

$$
\begin{aligned}
i_{L} & =0 \\
i_{C_{o}} & =-\frac{\hat{v}_{o}}{R_{L}} .
\end{aligned}
$$

From Fig. 6 , it is clear that $D_{\mathrm{a}}+D_{\mathrm{b}}+D_{\mathrm{c}}=1 / 2$, and $i_{\mathrm{L}}(B)=0$. Using the preceding relationships

$$
D_{b}=-\frac{\left(V_{i n}+\hat{v}_{o}\right)}{\left(V_{\text {in }}-\hat{v}_{o}\right)-\hat{v}_{o} D_{a} T_{s} r_{\text {loss }} / L} D_{a} .
$$

Hence,

$$
\overline{i_{c_{o}}}=-\frac{2 D_{a}^{2} T_{s}}{L} \frac{\left(V_{\text {out }}+V_{\text {in }}\right)\left(2 V_{\text {in }} V_{\text {out }}-V_{\text {out }}{ }^{2} D_{a} T_{s} r_{\text {loss }} / 2 L\right)}{\left(V_{\text {out }}-V_{\text {in }}+V_{\text {out }} r_{\text {loss }} D_{a} T / L\right)\left(2+D_{a} T_{s} r_{\text {loss }} / L\right)}-\frac{\hat{v}_{o}}{R_{L}} .
$$

The results of static characteristics are obtained by letting $d \widehat{v}_{o} / d t=0$, therefore

$P_{o}=\frac{2\left(D_{a} T_{s}\right)^{2}}{T_{s} L} \frac{\left(V_{\text {out }}+V_{\text {in }}\right)\left(2 V_{\text {in }} V_{\text {out }}-V_{\text {out }}{ }^{2} D_{a} T_{s} r_{\text {loss }} / 2 L\right)}{\left(V_{\text {out }}-V_{\text {in }}+V_{\text {out }} r_{\text {loss }} D_{a} T / L\right)\left(2+D_{a} T_{s} r_{\text {loss }} / L\right)}$

Using $D_{\mathrm{a}} T_{\mathrm{s}}=\varphi$

$P_{o}=\frac{2 \varphi^{2}}{T_{s} L} \frac{\left(V_{\text {out }}+V_{\text {in }}\right)}{\left(V_{\text {out }}-V_{\text {in }}+V_{\text {out }} r_{\text {loss }} \varphi / L\right)} \frac{\left(2 V_{\text {in }} V_{\text {out }}-V_{\text {out }}^{2} \varphi r_{\text {loss }} / 2 L\right)}{\left(2+\varphi_{s} r_{\text {loss }} / L\right)}$.

$B$ is calculated as

$$
B=\left(D_{a}+D_{b}\right) T_{s}=\frac{v_{o}\left(2+r_{\text {loss }} D_{a} T / L\right)}{\left(v_{o}-v_{i}\right)+v_{o} r_{\text {loss }} D_{a} T / L} \varphi
$$




\section{EXPERIMENTAL RESULTS}

In order to select the value of $r_{\text {loss, }}$, we perform some experiments with the prototype circuit. The main circuit is DAB dc-dc converter without additional circuits like snubber circuit. We had closed-loop-operation experiments with DSP TI TMS320F28335. And, also the value of A and B are manually supplied in this experiment. Experimental parameters are shown in Table I. Dead time of each switch is set as $1 \mu$ s.

\section{A. Power Efficiency}

Fig. 13 shows the power efficiency results for the both of the conventional and the proposed operation. It can be seen that the power efficiency of buck mode can be apparently improved by up to $37 \%$ using the proposed operation at $100 \mathrm{~W}$ as shown in Fig. 13 (a). It can be seen that the power efficiency of boost mode can be apparently improved by up to $30 \%$ at $100 \mathrm{~W}$ as shown in Fig. 13 (b).

\section{B. Estimating the Value of Loss}

Fig. 14 shows $\varphi-P_{\mathrm{o}}$ of analysis and experimental results. The value of $r_{\text {loss }}$ for the conventional operation is for the analysis is set to $2.0 \Omega$. The value is the measurement result of series resistance $r_{\mathrm{s}}$ of the transformer as shown in Table I measured with LCR meter Agilent 4263B.

The value of $r_{\text {loss }}$ for the proposed operation is calculated with averaged equivalent resistance with the averaged power calculation describe below.

$$
\begin{aligned}
\bar{P}_{\text {loss_conv }} \approx r_{s} & \frac{\int_{0}^{T}\left(i_{L m}(t)\right)^{2} d t}{T}=r_{s} \frac{V_{\text {in }}{ }^{2} T^{2}}{3 L^{2}} \\
\bar{P}_{\text {loss_proposed }} & \approx \frac{\int_{0}^{T} r_{s}\left(i_{L m}(t)\right)^{2} d t}{T}=\frac{r_{s} V_{\text {in }}^{2}}{T L^{2}} \int_{0}^{D T} t^{2} d t \\
& =\frac{r_{s} V_{\text {in }}^{2} D^{3} T^{2}}{3 L^{2}}=r_{s} D^{3} \frac{V_{\text {in }}^{2} T^{2}}{3 L^{2}}
\end{aligned}
$$

where $D$ is the conduction time ratio of switching term in no load condition and $T$ is the half of switching term.

From the calculation results, $r_{\text {loss }}$ of the proposed operation is calculated as the averaged equivalent resistance as

$$
r_{\text {loss }}=D^{3} r_{s} \text {. }
$$

From the result of our optional experiment, $D$ is obtained as $0.5 \mathrm{ohm}$. Therefore, the $r_{\text {loss }}$ for the proposed operation is set as $0.25 \mathrm{ohm}$.

Comparing loss including analysis and experimental results, the root mean square was in $4 \%$ both of boost mode and

\begin{tabular}{|c|c|c|}
\hline Item & Symbol & Specification \\
\hline \multicolumn{3}{|l|}{ Transformer } \\
\hline 1) Turns ratio & $A$ & $1: 1$ \\
\hline 2) Leakage inductance_(primary-referred) & $L$ & $110 \mu \mathrm{H}$ \\
\hline 3) Series resistance_(primary-referred) & $r_{s}$ & $2 \Omega$ \\
\hline \multicolumn{3}{|l|}{ Converter } \\
\hline 1) Rated output power & $P_{\mathrm{o}}$ & $1 \mathrm{~kW}$ \\
\hline 2) Rated input direct voltage & $V_{\text {in }}$ & $150 \mathrm{~V}$ \\
\hline 3) Rated output direct voltage & $V_{\text {out }}$ & $150 \mathrm{~V}$ \\
\hline 4) Switching frequency & $f_{\mathrm{s}}$ & $20 \mathrm{kHz}$ \\
\hline $\begin{array}{l}\text { 5) Absolute maximum ratings of } \\
\text { IGBT collector-emitter }\end{array}$ & $v_{\mathrm{CE}}$ & $600 \mathrm{~V}$ \\
\hline 6) On resistance of IGBT & $r_{\mathrm{t}}$ & $50 \mathrm{~m} \Omega$ \\
\hline 7) Absolute maximum ratings of diode & $i_{\mathrm{F}}$ & $30 \mathrm{~A}$ \\
\hline 8) Forward voltage of diode & $v_{\mathrm{F}}$ & $0.8 \mathrm{~V}$ \\
\hline 9) Recovery time of diode & $t_{\mathrm{rr}}$ & $0.1 \mu \mathrm{s}$ \\
\hline
\end{tabular}
buck mode.
TABLE I

SPECIFICATION OF DAB DC-DC CONVERTER

\section{CONCLUSION}

By the analysis of the circuit operation and the some experiments, the validation of the proposed operation for DAB dc-to-dc converter is revealed. Form the analysis, $P_{\mathrm{o}}$ can be calculated with the loss included analysis for both of the conventional and the proposed technique. The analysis results are well matched with the experimental results. Applying the two modes which are proposed operation in light load and conventional operation in heavy load, the circuit can be operated in the full load range. $37 \%$ maximum power efficiency improvement can be confirmed at light load.

\section{REFERENCES}

[1] R. Morrison, M. G. Egan, "A new power-factor-corrected singletransformer UPS design," IEEE Trans. Ind. Appl., vol 36, no.1, pp.171179, Jan./Feb. 2000.

[2] Florian Krismer, and Johann W. Kolar, "Accurate power loss model derivation of a high-current dual active bridge converter for Automotive Application," IEEE Trans. Ind. Electron., vol 57, no.3, pp.881-891, Mar. 2010.

[3] Huang-Jen Chiu and Li-Wei Lin, "A bidirectional dc-dc converter for fuel cell electric vehicle driving system," IEEE Trans. Power Electron., vol. 21, no. 4, pp. 950-958, Jul. 2007.

[4] F. Krismer, J. W. Kolar, "Accurate small-signal model for the digital control of an automotive bidirectional dual active bridge," IEEE Trans. Power Electron., vol. 24, no. 12, pp. 2756-2768, Dec. 2009.

[5] S. Inoue and H. Akagi, "A bidirectional dc-dc converter for an energy storage system with galvanic isolation," IEEE Trans. Power Electron. vol. 22, no. 6, pp. 2299-2306, Nov. 2007.

[6] Toshiro Hirose, Keisuke Nishimura, Takayuki Kimura, and Hirofumi Matsuo, "An AC-link Bidirectional DC-DC Converter with Synchronous Rectifier," in Proc. IECON, Nov. 2010. 


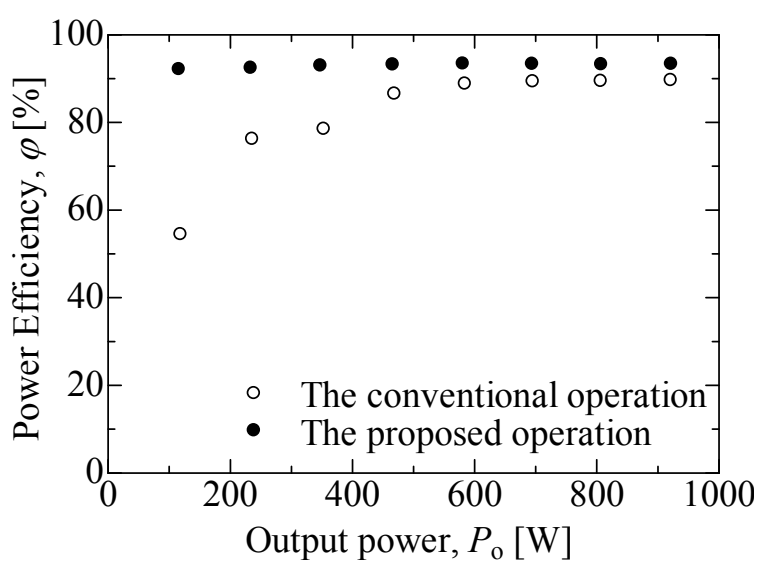

(a) Buck mode

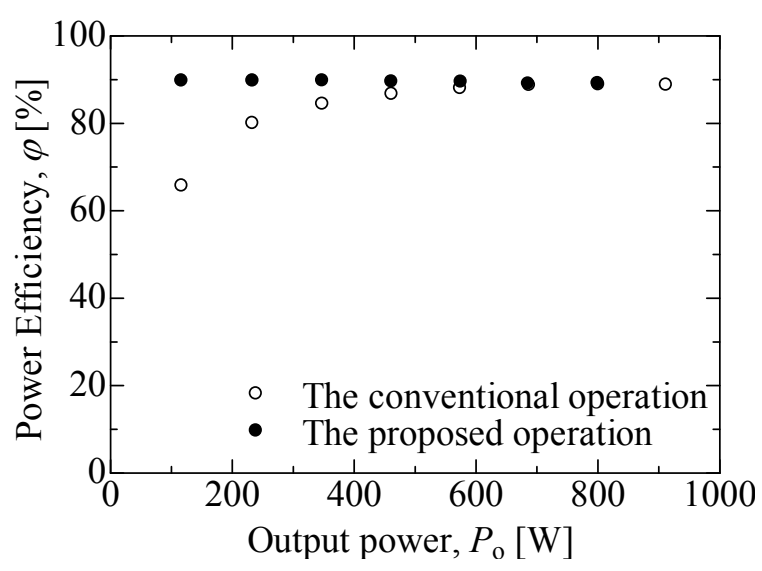

(b) Boost mode

Fig. 13. Power efficiency: (a) Buck mode $\left(V_{\text {in }}=200 \mathrm{~V}, V_{\text {out }}=150 \mathrm{~V}\right)$; (b) Boost $\operatorname{mode}\left(V_{\text {in }}=100 \mathrm{~V}, V_{\text {out }}=150 \mathrm{~V}\right)$.

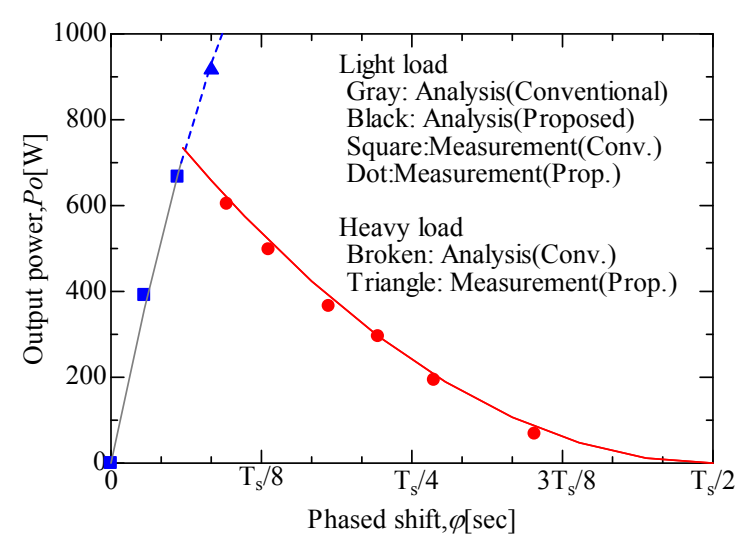

(a) Buck mode

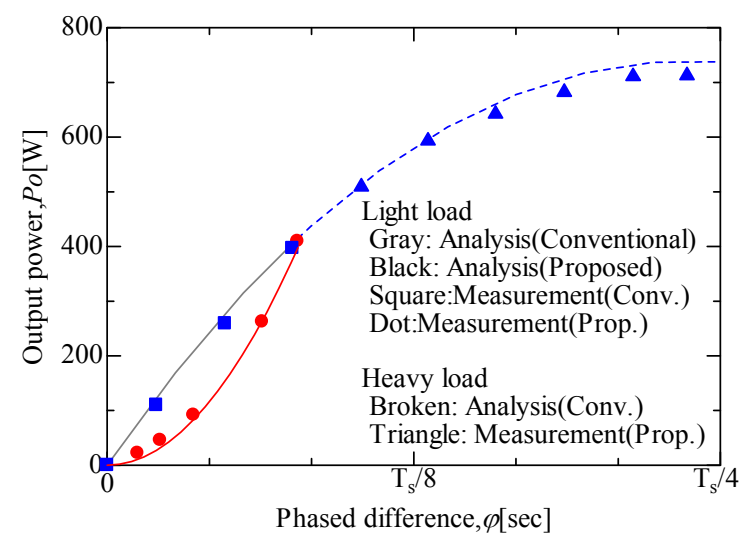

(b) Boost mode

Fig. 14. $\varphi-P_{\mathrm{o}}$ (analysis and experimental results).

[7] Mustansir H. Kheraluwala, Randal W. Gascoigne, Deepakraj M. Divan, and Eric D. Baumann, "Performance characterization of a high-power dual active bridge dc-to-dc converter," IEEE Trans. Industry Applications, vol.28, NO.6, pp. 1294-1301, Nov./ Dec. 1992.

[8] Shigenori Inoue and Hirofumi Akagi, "A bidirectional isolated dc-dc converter as a core circuit of the next-generation medium-voltage power conversion system, " IEEE Trans power Electron., vol.22, no.2, pp. 535-542, Mar. 2007.

[9] M. Pavlovsky, S. W. H. de Hann, and J. A. Ferreira, "Concept of 50kW DC/DC converter based on ZVS, quasi-ZCS topology and integrated thermal and electronic design," 2005 European Conference on Power Electronics and Applications.

[10] Haihua Zhou, A. M. Khambadkone, "Hybrid modulation for dualactive-bridge bidirectional converter with extended power range for ultracapacitor application," IEEE Trans. Ind. Appl., vol 45, no.4, pp.1434-1442, July/Aug. 2009.

[11] Yujin Song, P. N. Enjeti, "A new soft switching technique for bidirectional power flow, full-bridge DC-DC converter," in Proc. $37^{\text {th }}$ IAS Annual Meeting, pp.2314-2319, Oct. 2002.

[12] German G. Oggier, Guillermo O. Garcia, and Alejandro R. Oliva, "Switching control strategy to mimimize dual active bridge converter losses," IEEE Trans. Power Electron., vol 24, no.7, pp.1826-1838, July. 2009.
[13] Mika Takasaki, Yoichi Ishizuka, Tamotsu Ninomiya, Yutaka Furukawa, and Toshiro Hirose, "A Power Efficiency Improvement Technique for A Bi-Directional Dual Active Bridge DC-DC Converter at light load," EPE-ECCE 2013, pp.1-10, Sept. 2013.

[14] Rik W. A. A. De Doncker, Deepkraj M. Divan, and Mustansir H. Kheraluwala, "A Three-Phase Soft-Switched High-Power-Density $\mathrm{dc} / \mathrm{dc}$ Converter for High-Power Applications," IEEE Trans. Industry Applications, vol.27, NO.1, pp.63-73, Jan. / Feb. 1991.

[15] Shun Nagata, Mika Takasaki, Kazuhide Domoto, Toshiro Hirose , and Yoichi Ishizuka, "A static characteristic analysis and high efficient technique of the light load condition of Bi-Directional Dual Active Bridge DC-DC Converter," IEICE, , vol. 113, no. 155, EE2013-14, pp. 67-72, July 2013.

[16] Tamotsu Ninomiya, Masatoshi Nakahara, Toru Higashi, and Koosuke Harada, "A Unified Analysis of resonant Converters," IEEE Trans. Industry Applications, vol.27, NO.1, pp.63-73, Jan. / Feb. 1991. 